\title{
A class of Weighted Weibull Distribution
}

\author{
Saman Shahbaz \\ Department of Mathematics \\ COMSATS Institute of IT \\ Lahore, Pakistan \\ samans@ciitlahore.edu.pk \\ Muhammad Qaiser Shahbaz \\ Department of Mathematics \\ COMSATS Institute of IT \\ Lahore, Pakistan \\ qshahbaz@gmail.com \\ Nadeem Shafique Butt \\ College of Statistical and Actuarial Sciences \\ University of the Punjab \\ Lahore, Pakistan \\ nadeemshafique@hotmail.com
}

\begin{abstract}
The weighted Weibull model is proposed following the method of Azzalini (1985). Basic properties of the distribution including moments, generating function, hazard rate function and estimation of parameters have been studied.
\end{abstract}

Key Words: Weibull distribution, hazard function, moments, likelihood function.

\section{Introduction}

The Weibull distribution has been a powerful probability distribution in reliability analysis. The distribution generalizes the famous Exponential distribution and hence provides much wider applicability as compared with the exponential distribution. The Weibull distribution can also be used as an alternative to Gamma and Log-normal distribution in reliability engineering and life testing. Various extensions of Weibull and exponential distribution have been proposed in literature. An extension of exponential distribution has been provided by Nadarajah and Kotz (2005) using the logit of Beta distribution. Gupta and Kandu (1999) proposed a generalized exponential distribution which provides an alternative to exponential and Weibull distributions. The logit of Beta distribution has also been used by Famoye et al (2005) to introduce the Beta-Weibull distribution alongside its major properties. The exponentiated Weibull distribution has been extensively studied by Mudholkar and Srivastava (1993) as an extended model for modeling of bathtub data. The distribution provides classical Weibull and exponential distributions and special case. Madholkar et al. (1996) 
introduced the generalized Weibull distribution for modeling of survival time data. The proposed distribution again provides several standard distributions as special case. Recently Gupta and Kundu (2009) proposed a weighted exponential distribution by using the method of Azzalini (1985). The proposed model can be used as an alternative to Gamma and Weibull distribution. Using the same idea of Azzalini (1985) we proposed the weighted Weibull distribution in the following section. Some standard properties are studied in section 3 and parameter estimation has been done in section 4 of the paper.

\section{The Weighted Weibull Distribution}

Azzalini (1985) proposed a method of obtaining weighted distributions from independently identically distributed (i.i.d.) random variables. The proposed family of distributions uses density function of one random variable and distribution function of other random variable. To simplify the idea suppose two random variables $X_{1}$ and $X_{2}$ are i.i.d. random variables with distribution function $F(x)$. Azzalini (1985) suggested that a weighted class of density functions can be obtained by using:

$$
f_{X}(x)=\frac{1}{P\left(\alpha X_{1}>X_{2}\right)} f_{Y}(x) F_{Y}(\alpha x) ; \alpha>0 .
$$

Gupta and Kundu (2009) uses (1) to propose the weighted exponential distribution. We propose the weighted Weibull distribution by slightly modifying (1) as:

$$
f_{X}(x)=\frac{1}{P\left(\alpha^{1 / \beta} X_{1}>X_{2}\right)} f_{Y}(x) F_{Y}\left(\alpha^{1 / \beta} x\right) ; \alpha, \beta>0 .
$$

Now when $X_{1}$ and $X_{2}$ are i.i.d. as Weibull random variable with shape parameter $\beta$ and scale parameter $\lambda$ then using $f_{Y}(x)=\lambda \beta x^{\beta-1} \exp \left(-\lambda x^{\beta}\right)$ and $F_{Y}(x)=1-\exp \left(-\lambda x^{\beta}\right)$ in (2) we obtain following weighted Weibull distribution:

$$
f_{X}(x)=\frac{\alpha+1}{\alpha} \lambda \beta x^{\beta-1} \exp \left(-\lambda x^{\beta}\right)\left\{1-\exp \left(-\alpha \lambda x^{\beta}\right)\right\} ; \quad \alpha, \beta, \lambda, x>0 .
$$

The weighted exponential distribution of Gupta and Kundu (2009) can be immediately obtained from (3) by using $\beta=1$. The distribution (3) can be used for modeling of life time data with much more flexibility. The plot of density function (3) for $\lambda=1$ and for various choices of $\alpha$ and $\beta$ is given below: 
Figure 1: Plot of density function of Weighted Weibull Distribution

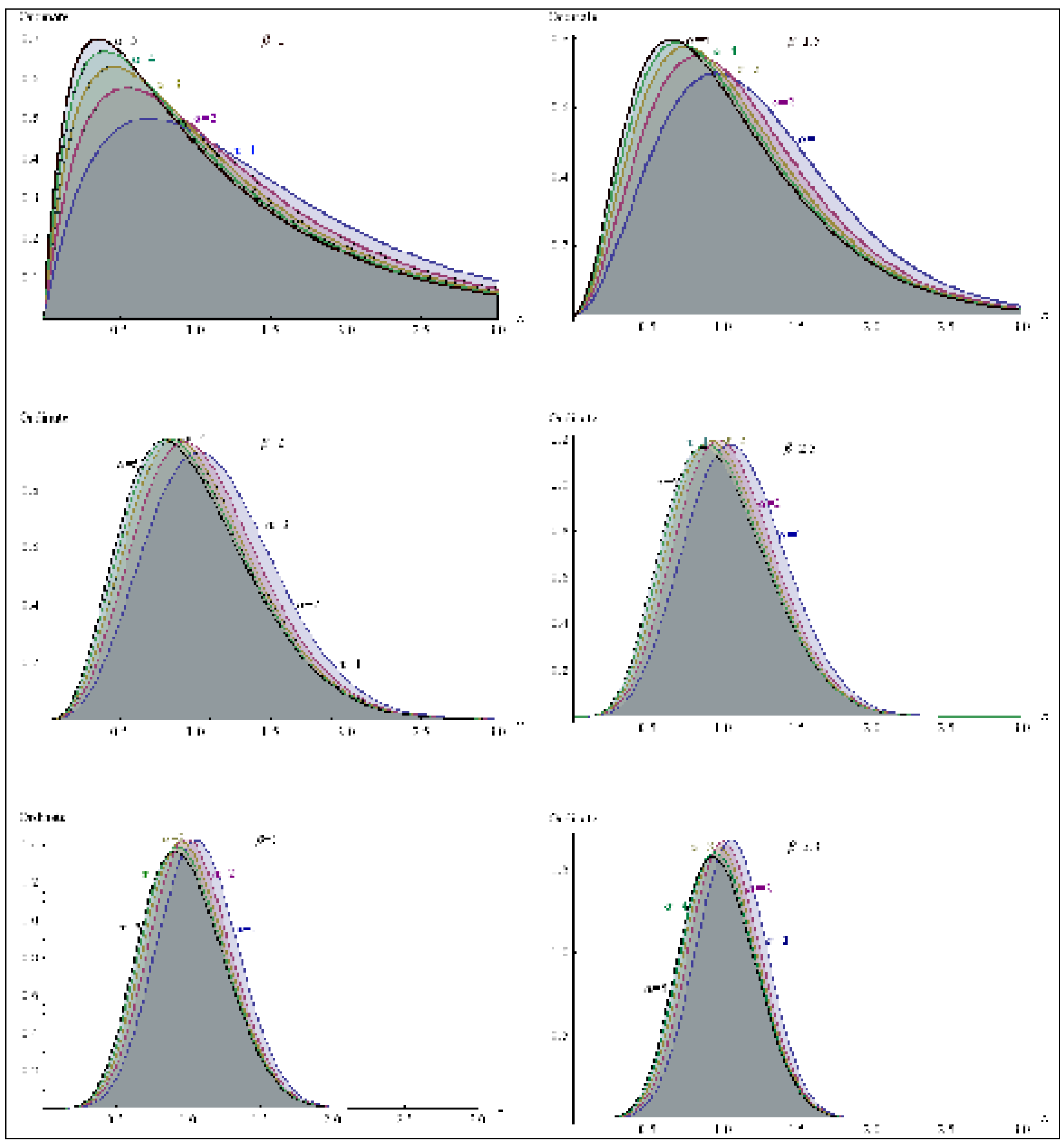

The plot of density shows that for small values of $\beta$ the distribution is positively skewed. The distribution approaches towards symmetry as value of $\beta$ increases. The scale parameter $\lambda$ can be loosely taken as 1 for sake of simplicity.

We now present some common properties of distribution (3) in following section. 


\section{Properties}

We have defined the weighted Weibull distribution in (3). The distribution function of (3) is given as:

$$
\begin{aligned}
F_{X}(x) & =\int_{0}^{x} f(w) d w \\
& =\int_{0}^{x} \frac{\alpha+1}{\alpha} \beta w^{\beta-1} \exp \left(-w^{\beta}\right)\left\{1-\exp \left(-\alpha w^{\beta}\right)\right\} d w ;
\end{aligned}
$$

After simplification, the distribution function is:

$$
F_{X}(x)=\frac{\alpha+1}{\alpha}\left[\left\{1-\exp \left(-x^{\beta}\right)\right\}-\frac{1}{\alpha+1}\left\{1-\exp \left(-(1+\alpha) x^{\beta}\right)\right\}\right] .
$$

Using (3) and (4), the hazard rate function of weighted Weibull distribution is:

$$
h(x)=\frac{f_{X}(x)}{1-F_{X}(x)}=\frac{\beta(1+\alpha) x^{\beta-1}\left\{1-\exp \left(-\alpha x^{\beta}\right)\right\}}{1+\alpha-\exp \left(-\alpha x^{\beta}\right)} .
$$

The plot of hazard rate function for various choices of $\alpha$ and $\beta$ is given in figure 2. The plot shows that hazard rate has increasing trend for all values of $\alpha$ and $\beta$ considered for plotting function (5). The hazard rate function given in (5) has decreasing trend when $\beta<1$ and so the distribution (3) has wide spread applicability in studying life time of components.

The moment generating function of density (3) can readily obtained as:

$$
\begin{aligned}
M_{X}(t) & =E e^{t x}=\int_{0}^{\infty} e^{t x} f_{X}(x) d x \\
& =\int_{0}^{\infty} e^{t x} \frac{\alpha+1}{\alpha} \beta x^{\beta-1} \exp \left(-x^{\beta}\right)\left\{1-\exp \left(-\alpha x^{\beta}\right)\right\} d x ;
\end{aligned}
$$

After simplification, the moment generating function is readily obtained as:

$$
M_{X}(t)=\sum_{j=0}^{\infty} \frac{t^{j}}{j ! \alpha}\left\{1+\alpha-(1+\alpha)^{-j / \beta}\right\} \Gamma\left(1+\frac{j}{\beta}\right) .
$$

The $r$-th moment can be immediately written from (6) as:

$$
\mu_{r}^{\prime}=E\left(X^{r}\right)=\frac{1}{\alpha}\left\{1+\alpha-(1+\alpha)^{-r / \beta}\right\} \Gamma\left(1+\frac{r}{\beta}\right) .
$$

Mean and variance of weighted Weibull distribution can be found by using (7). We can see from (7) that the moments exist for all integer values of $r$. 


\section{Figure 2: Hazard Rate Function of Weighted Weibull Distribution}
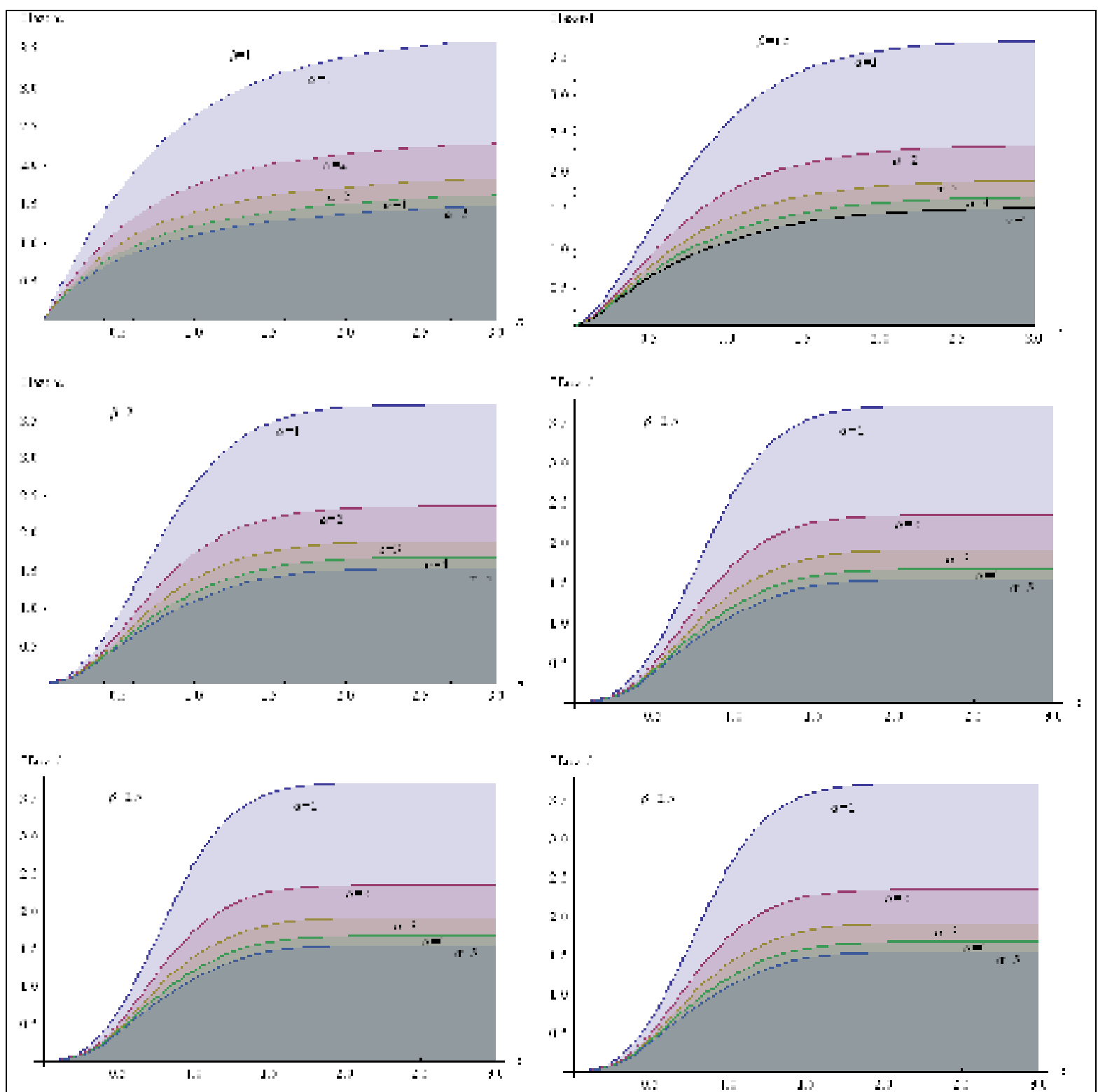

\section{Parameter Estimation}

The density function of weighted Weibull distribution is given in (3). The likelihood function for a sample of size $n$ can be immediately written as:

$$
L\left(x_{1}, \ldots, x_{n} \mid \alpha, \beta\right)=\left(\frac{\alpha+1}{\alpha}\right)^{n} \beta^{n} \prod_{i=1}^{n} y_{i}^{\beta} \exp \left(-\sum_{i=1}^{n} y_{i}^{\beta}\right) \prod_{i=1}^{n}\left\{1-\exp \left(-\alpha y_{i}^{\beta}\right)\right\}
$$

The logarithm of (8) is:

$$
\ln L=n \ln \left(\frac{\alpha+1}{\alpha}\right)+n \ln \beta+\sum_{i=1}^{n}(\beta-1) \ln y_{i}-\sum_{i=1}^{n} y_{i}^{\beta}+\sum_{i=1}^{n} \ln \left\{1-\exp \left(-\alpha y_{i}^{\beta}\right)\right\}
$$


Partial derivatives of (9) w.r.t. the unknown parameters $\alpha$ and $\beta$ are:

$$
\begin{aligned}
& \frac{\partial \ln L}{\partial \alpha}=-\frac{n}{\alpha}+\frac{n}{\alpha+1}+\sum_{i=1}^{n} \frac{y_{i}^{\beta} \exp \left(-\alpha y_{i}^{\beta}\right)}{1-\exp \left(-\alpha y_{i}^{\beta}\right)} \\
& \frac{\partial \ln L}{\partial \beta}=\frac{n}{\beta}+\sum_{i=1}^{n} \ln y_{i}-\sum_{i=1}^{n} y_{i}^{\beta} \ln y_{i}+\sum_{i=1}^{n} \frac{\alpha y_{i}^{\beta} \ln y_{i} \exp \left(-\alpha y_{i}^{\beta}\right)}{1-\exp \left(-\alpha y_{i}^{\beta}\right)}
\end{aligned}
$$

The estimating equations for unknown parameters are:

$$
\begin{aligned}
& -\frac{n}{\alpha}+\frac{n}{\alpha+1}+\sum_{i=1}^{n} \frac{y_{i}^{\beta} \exp \left(-\alpha y_{i}^{\beta}\right)}{1-\exp \left(-\alpha y_{i}^{\beta}\right)}=0 \\
& \frac{n}{\beta}+\sum_{i=1}^{n} \ln y_{i}-\sum_{i=1}^{n} y_{i}^{\beta} \ln y_{i}+\sum_{i=1}^{n} \frac{\alpha y_{i}^{\beta} \ln y_{i} \exp \left(-\alpha y_{i}^{\beta}\right)}{1-\exp \left(-\alpha y_{i}^{\beta}\right)}=0
\end{aligned}
$$

The unknown parameters can be obtained by solving (10) and (11) iteratively.

\section{Numerical Example}

In this section we present a numerical example to show the advantage of proposed distribution over available distributions in literature. The data below shows life length of components in years.

\begin{tabular}{|l|c|c|c|c|c|c|}
\hline Life & $0.0-1.0$ & $1.0-2.0$ & $2.0-3.0$ & $3.0-4.0$ & $4.0-5.0$ & $>5.0$ \\
\hline Frequency & 67003 & 56554 & 44 & 13 & 4 & 1 \\
\hline
\end{tabular}

We have fitted the classical Weibull distribution with $\beta=3.0$, weighted Exponential distribution of Gupta and Kundu (2009) with $\alpha=4.0$ and the weighted Weibull distribution with $\alpha=4.0$ and $\beta=3.0$. The computed value of $\chi_{0}^{2}$ for three distributions; $p$-value in parenthesis; turned out to be $4327.773 \quad(<0.0001)$, $32935.274(<0.0001)$ and $3.300(0.192)$. The analysis clearly shows that our proposed weighted Weibull distribution fits the observed data reasonably well as compared with other competing models.

\section{References}

1. Azzalini, A. (1985). A class of distributions which include the normal ones, Scand. J. Stat., Vol. 12, 171-178.

2. Famoye, F., Lee, C. and Olumolade, O. (2005). The Beta-Weibull Distribution, Journal of Statistical Theory and Applications, Vol. 4(2), 121-136.

3. Gupta, R. D. and Kundu, D. (1999). Generalized exponential distribution, Aust. N. Z. J. Stat. Vol. 41, 173-188. 
4. Gupta, R. D. and Kundu, D. (2009). A new class of weighted exponential distributions, Statistics, 43(6), 621-634.

5. Mudholkar, G. S. and Srivastava, D. (1993). Exponentiated Weibull family for analyzing bathtub failure-rate data, IEEE Trans. on Reliab., Vol. 42(22), 299-302.

6. Mudholkar, G.S., Srivastava D.K. and Kollia G.D. (1996). A Generalization of the Weibull Distribution with Application to the Analysis of Survival Data, Journal of the American Statistical Association, Vol. 91(436), 1575-1583.

7. Nadarajah, S., Kotz, S. (2005). The Beta-Exponential Distribution, Reliability Engineering and System Safety, Vol. 91, 689-697. 